\title{
A Bioinspired Airfoil Optimization Technique Using Nash Genetic Algorithm
}

\author{
Hamid Isakhani $^{1}$, Caihua Xiong ${ }^{2}$, Shigang Yue ${ }^{1}$, and Wenbin Chen ${ }^{2}$
}

\begin{abstract}
Natural fliers glide and minimize wing articulation to conserve energy for endured and long range flights. Elucidating the underlying physiology of such capability could potentially address numerous challenging problems in flight engineering. However, primitive nature of the bioinspired research impedes such achievements, hence to bypass these limitations, this study introduces a bioinspired non-cooperative multiple objective optimization methodology based on a novel fusion of PARSEC, Nash strategy, and genetic algorithms to achieve insect-level aerodynamic efficiencies. The proposed technique is validated on a conventional airfoil as well as the wing crosssection of a desert locust (Schistocerca gregaria) at low Reynolds number, and we have recorded a $77 \%$ improvement in its gliding ratio.
\end{abstract}

\section{INTRODUCTION}

Natural flight has been one of man's long-standing interests since the beginning of time. After Leonardo da Vinci's early drawings of an ornithopter in 1485, Rayleigh [1] was perhaps the first to demonstrate a satisfactory report on soaring flight of birds, followed by Walker [2] quantitatively explaining birds' flapping flight, and later Ellington's elaborate contributions on the aerodynamics of hovering insect flight [3].

Among other insects, locusts possess tandem wings with concealed characteristics extremely favorable in addressing aeronautical challenges. They distinctly inspire this research due to; their long-range flight and endured gliding capabilities [4], and their major $(>70 \%)$ neuron dedication to collision avoidance and coordinated flight [5]-[7], proving them as excellent but under-appreciated natural aviators. Indeed these traits have compelled researchers to study live locusts since the mid-20c. Weis-Fogh [8], among others, reviewed the biology and aerodynamics of locusts under steady flight conditions. And Cloupeau et al. [9] determined the instantaneous lift and the wing deformation of locusts using high-speed filming.

Despite the tremendous contributions, we are yet to elucidate this insect's great potential to conserve energy on distant flights by gliding for such long intervals of time [4], [10], [11]. Energy conservation is an extremely important factor for a natural flier which is best achieved by reducing the flapping of wings, thus, gliding. It is reported that swarm of locusts cross the Red Sea strait regularly. Also during the 1987-89 plague, locusts crossed the Atlantic Ocean in

\footnotetext{
${ }^{1}$ Computational Intelligence Laboratory (CIL), School of Computer Science, University of Lincoln, LN6 7TS, United Kingdom hIsakhanielincoln.ac.uk

${ }^{2}$ The State Key Laboratory of Digital Manufacturing Equipment and Technology, Huazhong University of Science and Technology, Wuhan, 430074, China. chxiong@hust. edu. cn
}

ten days [4]. This flight range on such a small sized flier can only be achieved by an extreme energy conservation regimen defined by a high aerodynamic efficiency (lift-drag ratio) famously exhibited by locusts.

Continuous attempts are being made to enhance our knowledge of locust gliding and its aerodynamic footprint [11]-[14], however, due to the limitations and the intricacies involved in this process, we are yet to achieve such high aerodynamic efficiencies. Thus, alternatively this study proposes an enhancement of the current findings by performing a mathematical geometry optimization in order to alleviate the shortcomings of the biological deciphering. Although some studies suggest methodologies such as; extracting energy in pitching and plunging tandem wings to endure flight [15], [16], or perhaps combining wing kinematics (flexibility) and morphology to improve the aerodynamic performance [17], [18], yet a systematic approach ceases to exist that could potentially yield the desired lift-drag ratio.

Primarily, there is a requirement for a parameterization scheme that can transform the chordwise cross-section profile of the locust wing from geometrical to a precise mathematical form. Generally, there is a broad range of schemes that serve this purpose [19], B-splines, Bezier curves, and HicksHenne shape functions are some of the commonly implemented methods for conventional smooth-surfaced airfoils. However, due to the radical nature of our corrugated bioinspired airfoil, a readily available scheme may not perform satisfactorily. Hence, a rather flexible \& physically intuitive methodology such as the PARSEC parameterization [20], [21] is adapted here to express our airfoil as a linear combination of unknown base functions with 11 major attributes directly linked to the geometry (angle of incidence, abscissa, edge radius, thickness, etc.).

Furthermore, a suitable optimization technique is required such that; it forms an apt fusion with the parameterization scheme, simultaneously optimizing multiple functions noncooperatively as demanded in aerodynamics where the lift can only be maximized at the cost of drag. Hence, reaching a state of equilibrium to establish the best possible trade-off strategy between the two functions. Usually, standard Pareto Genetic Algorithms (GAs) are implemented for such multiple objective optimization problems introduced by Goldberg [22], which provides solutions to a wide range of applications as a result of further developments presented in [23]. Nevertheless, cooperative nature of the Pareto ranking is limited to the use of sharing or mating constraints. However, the GAs introduced by Nash [24] originating from Game Theory and Economics, tend to solve multiple objective 
optimization problems faster [25] non-cooperatively, that is highly favorable in our application where the design variables (DVs) pose well distinctions, and the evaluations are computation-intensive.

To conclude, this study explores the aerodynamic performance of locust wing in gliding mode using 2D computational fluid dynamics (CFD) simulations, whose results (obtained lift-drag ratio) serve as an input to our proposed mathematical optimization technique based on a novel fusion of PARSEC, Nash strategy, and genetic algorithms to acquire Nash equilibrium/optimized wing cross-section profiles with an initial condition set at $75 \%$ of the original PARSEC parameters i.e. the DVs are reduced by $25 \%$ in dimension. Finally, comparative studies are provided to draw conclusions on the literature, original, and the optimized aerodynamic performances obtained.

\section{WING MODEL}

Specimen studied here are the wings of six typical farm bred female desert locusts Schistocerca gregaria procured from an insect farm in Cangzhou, China. Cyanoacrylate was sprayed on the wing-roots of locusts to hold the deployed (gliding) fore- and hindwings erected for sectioning at $20 \%$, $40 \%, 60 \%$, and $80 \%$ spanwise chord. Wing sections having variable thickness of 2-3 $\mu \mathrm{m}$ were carefully placed in a lightbox for a digital microscopic scanning using a Canon EOS 550D SLR camera on an AmScope 45x stereo microscope.

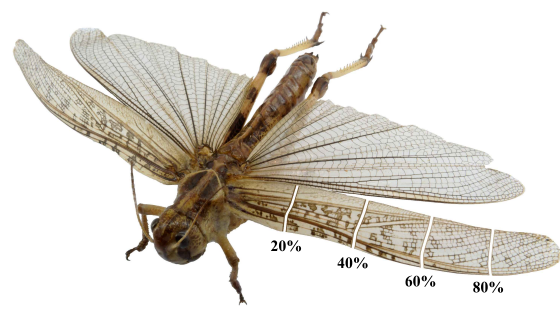

(a)

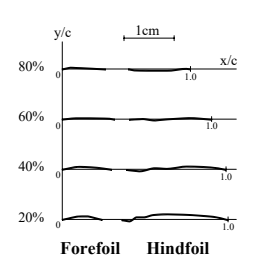

(b)
Fig. 1. Illustration of locust wing cross-section profiles at $20 \%, 40 \%, 60 \%$, and $80 \%$ spanwise chord from wing root. (a) dissected locust wing, and (b) digitized wing cross-section profiles

To validate our observations, wing profiles from the literature [11], [12] were compared, and it was concluded that the profiles presented by Walker et al. [12] in a smoke flow visualization experiment correlated with our recordings. Hence, the profiles shown in Figure 1(a) were confirmed and digitized using SolidWorks to generate igs files for the CFD simulations. Apart from the wing profiles, the process of digitization involved all three (front, top, and side) views of the wings prior to sectioning in order to facilitate a $3 \mathrm{D}$ reconstruction for our future study as well as a precise $2 \mathrm{D}$ reconstruction process involving every crucial detail such as fore- and hindwing transverse \& longitudinal spacing.

\section{A. Numerical Methods}

The ultimate objective of this section is to analyze the air flow around the locust airfoils in order to understand the influence of different factors on its aerodynamic performance.
To achieve this, the wings in a computational domain are simulated using computer-aided design (CAD) and numerical analysis software solutions; Dassault Systémes SolidWorks ${ }^{\circledR}$ and ANSYS ${ }^{\circledR}$ Workbench, respectively. Figure 1(b) shows the 2D CAD profiles of the locust wing digitized in SolidWorks. The 2D CFD simulations are prepared as a platform to validate our proposed optimization technique and study its impact on bioinspired airfoils. Here, the airfoil sections are considered as rigid bodies gliding with a standardized thickness of $2 \mathrm{~mm}$ [11]. A locust like most other insects flies at subsonic flight regime, thus we can consider the nature of flow over its wings to be incompressible, turbulent, and our solver; pressure-based. The CFD analysis of a low Reynolds number flow field over an airfoil is governed by the 2D Navier-Stokes equation [26]. Ideally, the nature of flow in gliding flight must be laminar, however, in case of a natural flier the wing corrugations cause a mild turbulence [27] that we define by the Spalart-Allmaras model [28] which solves Reynolds-averaged Navier-Stokes equations.

Ultimately, the solver schemes and methodologies are set as $1^{\text {st }}$ order QUICK for spatial discretization and SIMPLEC method for solving pressure-velocity coupling. Additionally, a time step is also performed on iterations by two-step implicit expression law to facilitate temporal accuracy of the solutions.

\section{B. Discretization and Boundary Conditions}

For this study, a sufficiently large two-dimensional hybrid CO-type computational domain is created to encompass the airfoil with minimum wall influence on its flow field. To achieve this, domain shown in Figure 2 is designed to measure 25 and 20 times wing chord length in $x$ and $y$ direction, respectively. The air inflow condition is set at the domain inlet that is the edge facing airfoil leading-edge (LE), and the outflow condition is set at the domain outlet, facing the trailing-edge (TE) of the airfoil. These dimensions are strictly fixed to avoid any wall influence on the flow field, particularly on the turbulence generated by the wing corrugation. As mentioned earlier, due to locust's relatively low flight velocity, the Reynolds number used to study its gliding must remain below 10,000 [11], [29]. Hence, $R e$ is set within this range for all the CFD analysis presented in this work.

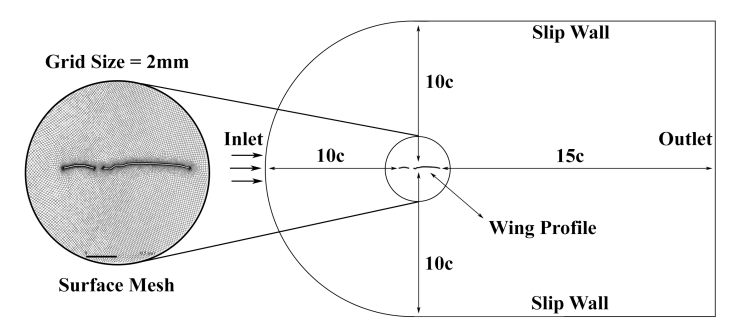

Fig. 2. Illustration of the simulation environment; high quality surface mesh generated in ANSYS Meshing for the locust wing cross section profile inside a CO-type computational domain with schematic representation and boundary conditions setup. 'c' stands for wing chord.

Furthermore, the proposed domain is meshed with high 
quality surface grids in ANSYS Meshing. Figure 2 shows a part of the mesh generated for the airfoil at $20 \%$ spanwise chord with a global element size defined as $2 \mathrm{~mm}$, airfoil top and bottom surface divided into 200 elements each, and the leading and trailing edges as 10 parts, producing a converged lift and drag coefficients of 0.3475 and 0.0228 , respectively, at $R e=7000$ and $\alpha=2^{\circ}$. The finalized mesh resolution is validated against literature [11], and the obtained aerodynamic forces for different grid sizes. Where, smaller than $2 \mathrm{~mm}$ grids practically ceased to influence the solution convergence and the aerodynamic performance $(<1.8 \%$ difference in forces).

\section{Optimization Methodology}

In contrast to some of the methodologies demonstrated in the literature to improve bioinspired airfoils [11], [15], [18], we treat the locust wing cross-section profile as a conventional airfoil and hence, implement a systematic approach to mathematically optimize its aerodynamic performance. Here, we propose a fusion of the bioinspired Genetic Algorithms (GAs), Nash strategy, and PARSEC parameterization that has been validated against its peer models [25] in terms of computational efficiency and solution accuracy.

\section{A. Nash Genetic Algorithm}

Nash [24] presented a non-cooperative multiple objective optimization technique in the context of Economics and Game Theory. This approach introduces the notion of players denoting the number of objectives in a given optimization problem. It states that unilateral alteration of strategy does not bring any gain to any player individually [30]. For a two player scenario, a Nash equilibrium state $N$ is reached when no further improvement of criterion can be achieved by any of the players. This is mathematically expressed as,

$$
\left\{\begin{array}{l}
\text { Determine }\left(\bar{p}_{1}, \bar{p}_{2}\right) \in P_{1} \times P_{2} \text { such that } \\
f_{1}\left(\bar{p}_{1}, \bar{p}_{2}\right)=\min _{p_{1} \in P_{1}} f_{1}\left(p_{1}, \bar{p}_{2}\right) \text { and } \\
f_{2}\left(\bar{p}_{1}, \bar{p}_{2}\right)=\min _{p_{2} \in P_{2}} f_{2}\left(\bar{p}_{1}, p_{2}\right)
\end{array}\right.
$$

where $f_{1}, f_{2}$ are the player objective functions, while $\left(p_{1}, p_{2}\right) \in P_{1} \times P_{2}$ are the player strategies, defined in their local strategy domain. Specifically, these strategies are each a set of variables given as $p_{1}=\left[\gamma_{1} \ldots, \gamma_{n}\right], p_{2}=$ $\left[\eta_{1} \ldots, \eta_{m}\right]$ with a dimension $n, m$ that is defined by the airfoil parameterization scheme described later.

On the other hand, the Genetic Algorithms (GAs) are based on an adaptive heuristic search approach inspired by the concept of natural selection and genetics, where a population is permitted to evolve only after satisfying a certain set of selection rules that are aimed at maximizing an objective function (fitness) or minimizing a cost function [31].

We combine the two schemes to deduce a two-player Nash equilibrium game strategy [32], [33], that describes $X, Y$ as two players' variable sets, and $f_{1}, f_{2}$ as two real valued functions of $X, Y$ representing the players' cost/objective. According to Nash adjustment process [34], players set their

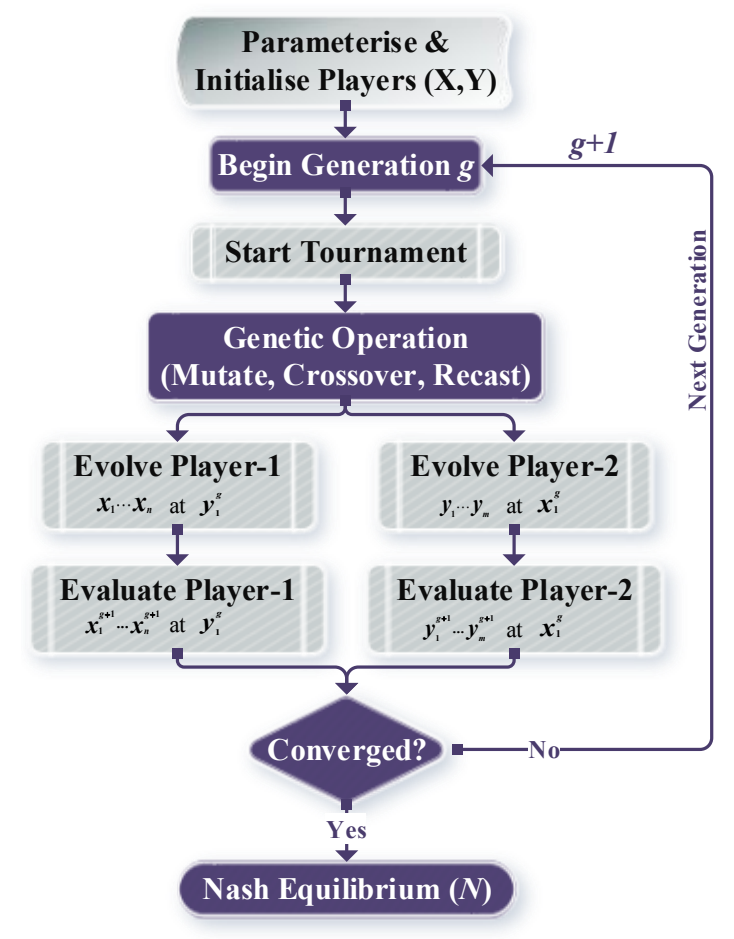

Fig. 3. The process flowchart for a Nash strategy based Genetic Algorithm.

outputs in turns, which must be their best response to the opponent's last output until convergence (otherwise known as the Nash equilibrium of the game). This solution is represented by a string or a chromosome (GA notion) given as $s=x, y$ where $x$ and $y$ are the subsets of variables belonging to players 1 and $2\left(p_{1}, p_{2}\right)$ within the metric space $X, Y$, respectively.

Therefore, Nash equilibrium can be defined as the optimization of $s$ by $p_{1}$ modifying $x$ with respect to the objective function $\left(f_{1}\right)$ while $y$ is fixed by $p_{2}$, and symmetrically $p_{2}$ optimizing the chromosome with respect to $f_{2}$ by changing $y$ while $x$ is fixed by $p_{1}$ [24]. Applying this procedure here as shown in Figure 3, player-1 optimizes $x^{g}$ using $y^{g-1}$ in order to evaluate the chromosome $\left(s=x^{g}, y^{g-1}\right)$, while simultaneously, player-2 is optimizing $y^{g}$ using $x^{g-1}$ to evaluate his chromosome $\left(s=x^{g-1}, y^{g}\right)$. In this algorithm, one random population of individuals is created for each player to perform the task of optimization for that player. The classification is based on the evaluation of a fitness function that considers the matching outcomes of individuals in population-1 with all the individuals in population-2, scoring 1 or -1 for a win or lose, respectively and 0 for a draw.

$$
\left\{\begin{array}{lll}
\text { if } & f_{1}\left(x_{i}^{g}, y^{g-1}\right)>f_{1}\left(x^{g-1}, y_{i}^{g}\right), & \text { fitness }=1 \\
\text { if } \quad f_{1}\left(x_{i}^{g}, y^{g-1}\right)<f_{1}\left(x^{g-1}, y_{i}^{g}\right), & \text { fitness }=-1 \\
\text { if } \quad f_{1}\left(x_{i}^{g}, y^{g-1}\right)=f_{1}\left(x^{g-1}, y_{i}^{g}\right), & \text { fitness }=0
\end{array}\right.
$$

And similarly, for player 2 (only $f_{1}$ changes to $f_{2}$ ). This facilitates a clear and precise sorting criterion based on which the individuals from population- 1 are sorted on $f_{1}$ and viceversa, for an equal fitness value. After the formation of 
a mating pool for the parent chromosome, crossover and mutations are performed on each player population. A mating pool for parent chromosome is generated and common GA techniques such as crossover and mutation are performed on each player population.

\section{B. Nash GA-PARSEC Fusion}

Parameterization is the foundation of an optimization process that is responsible for transforming a physical problem into a mathematical one by encoding a geometrical shape to form a number of variables. Here we adapt and implement

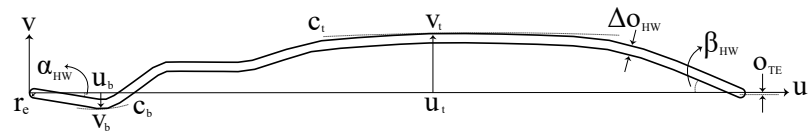

(a)

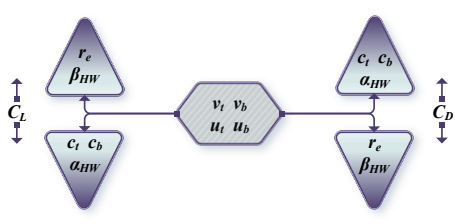

(b)

Fig. 4. Illustration of the Nash GA-PARSEC fusion and parameterization (a) PARSEC applied on locust hindfoil (b) dependency levels of player-1 $\left(C_{L}\right)$ and player-2 $\left(C_{D}\right)$ on different design variables.

a reliable airfoil-specific parameterization scheme developed by Sobieczky [21], that is based on the idea of expressing an airfoil geometry as a linear combination of unknown base functions with 11 major attributes assigned to the wellestablished airfoil shape parameters shown in Figure 4 and defined in Table I. These parameters are selected as the airfoil's control variables such that the ultimate shape shall be determined by solving a linear system.

TABLE I

LIST OF PARSEC PARAMETERS WITH CORRESPONDING DEFINITION

\begin{tabular}{lcc}
\hline PARSEC & GEOMETRY & DESCRIPTION \\
\hline$a_{1}$ & $r_{e}$ & edge radius \\
$a_{2}$ & $u_{t}$ & top crest horizontal position \\
$a_{3}$ & $v_{t}$ & top crest vertical position \\
$a_{4}$ & $c_{t}$ & top crest curvature \\
$a_{5}$ & $u_{b}$ & bottom crest horizontal position \\
$a_{6}$ & $v_{b}$ & bottom crest vertical position \\
$a_{7}$ & $c_{b}$ & bottom crest curvature \\
$a_{8}$ & $o_{T E}$ & trailing edge vertical offset \\
$a_{9}$ & $\Delta_{H W}$ & profile thickness \\
$a_{10}$ & $\alpha_{H W}$ & leading-edge angle of incidence \\
$a_{11}$ & $\beta_{H W}$ & trailing edge angle of incidence \\
\hline
\end{tabular}

As shown in Figure 4(b), players 1 and 2 are defined as the coefficients of lift and drag, respectively. Furthermore, these players are assigned with different physical Design Variables (DVs). Dependency levels of each player on a particular DV is illustrated in Figure 4(b), described as, $C_{L}$ being mainly influenced by the top crest curvature $\left(c_{t}\right)$, the lower crest curvature $\left(c_{b}\right)$, the TE vertical offset $\left(o_{T E}\right)$, and the TE angle of incidence $\left(\beta_{H W}\right) . C_{D}$ on the other hand, is mainly influenced by the LE radius $\left(r_{e}\right)$, profile thickness
$\left(\Delta o_{H W}\right)$, and LE angle of incidence $\left(\alpha_{H W}\right)$. And ultimately, both coefficients equally depend on the top crest horizontal and vertical positions $\left(u_{t}, v_{t}\right)$ as well as the bottom crest horizontal and vertical positions $\left(u_{b}, v_{b}\right)$. However, vertical offset of the trailing-edge $\left(o_{T E}\right)$ and the profile thickness $\left(\Delta o_{H W}\right)$ are not altered throughout this optimization process.

TABLE II

LIST OF NASH GA INPUT VARIABLE COMBINATIONS

\begin{tabular}{ccc}
\hline $\begin{array}{c}\text { Combination } \\
\text { (strategy) }\end{array}$ & $\begin{array}{c}\text { Player-1 }\left(C_{L}\right) \\
c_{t}, c_{b}, o_{T E}, \beta_{H W}\end{array}$ & $\begin{array}{c}\text { Player-2 }\left(C_{D}\right) \\
r_{e}, \Delta o_{H}, \alpha_{H W}\end{array}$ \\
\hline 1 & $v_{t}, u_{b}, v_{b}$ & $u_{t}$ \\
2 & $v_{t}, u_{b}$ & $u_{t}, v_{b}$ \\
3 & $v_{t}, v_{b}$ & $u_{t}, u_{b}$ \\
4 & $v_{t}$ & $u_{t}, u_{b}, v_{b}$ \\
5 & $u_{b}, v_{b}$ & $u_{t}, v_{t}$ \\
6 & $u_{b}$ & $u_{t}, v_{t}, v_{b}$ \\
7 & $v_{b}$ & $u_{t}, v_{t}, u_{b}$ \\
8 & - & $u_{t}, v_{t}, u_{b}, v_{b}$ \\
9 & $u_{t}, v_{t}, u_{b}, v_{b}$ & - \\
10 & $u_{t}, v_{t}, u_{b}$ & $v_{b}$ \\
11 & $u_{t}, v_{t}, v_{b}$ & $u_{b}$ \\
12 & $u_{t}, v_{t}$ & $u_{b}, v_{b}$ \\
13 & $u_{t}, u_{b}, v_{b}$ & $v_{t}$ \\
14 & $u_{t}, u_{b}$ & $v_{t}, v_{b}$ \\
15 & $u_{t}, v_{b}$ & $v_{t}, u_{b}$ \\
16 & $u_{t}$ & $v_{t}, u_{b}, v_{b}$ \\
\hline
\end{tabular}

To restrict the range of our analysis illustrated here, we prioritize the common Design Variables (cDVs) assignment to form 16 possible combinations determined by; $C_{n=4, p=2}=\sum_{i \in n_{0}} \frac{n !}{i !(n-1) !}=16$, where $n$ is the number of commonly influencing DVs $\left(u_{t}, v_{t}, u_{b}\right.$, and $\left.v_{b}\right), p$ is the number of players, and $n_{0}=[0, \ldots, n]$. These combinations are tabulated in Table II, where the highly influential DVs are assigned to their corresponding players, with different combinations of cDVs assigned to each player forming a total of 16 strategies. Furthermore, to simplify the process of evaluation we classify these strategies into five groups as; $4 \mathrm{~L}-0 \mathrm{D}$ for the combination with all the cDVs assigned to player-1, 3L-1D when $3 \mathrm{cDV}$ s are assigned to player-1 and 1 to player-2, 2L-2D when all the cDVs are shared equally among the players, 1L-3D for the case when $3 \mathrm{cDV}$ s belong to player-2 and one to player-1, and 0L-4D for the strategy where all the cDVs are assigned to player- 2 .

\section{RESUlts}

The proposed optimization process is performed on all the locust tandem wing cross-section profiles. However, the performance of the airfoil only at $20 \%$ spanwise chord is illustrated here for the sake of conciseness. The optimization results of a locust tandem wing cross-section profile at $20 \%$ spanwise chord is evaluated here as a sample case for the sake of concision. The fore- and hindfoils are parameterized separately but optimized together as a single profile to take their aerodynamic interactions into consideration, in contrast to the literature [11] where this crucial factor is neglected. The optimization process is performed at $75 \%$ of the original PARSEC parameters, without geometric \& aerodynamic constraints. Also it must be noted that the 
proposed procedure is not automated due to the radical DVs forming the corrugated geometry of the bioinspired airfoil, hence the process is manually operated and monitored caseby-case.

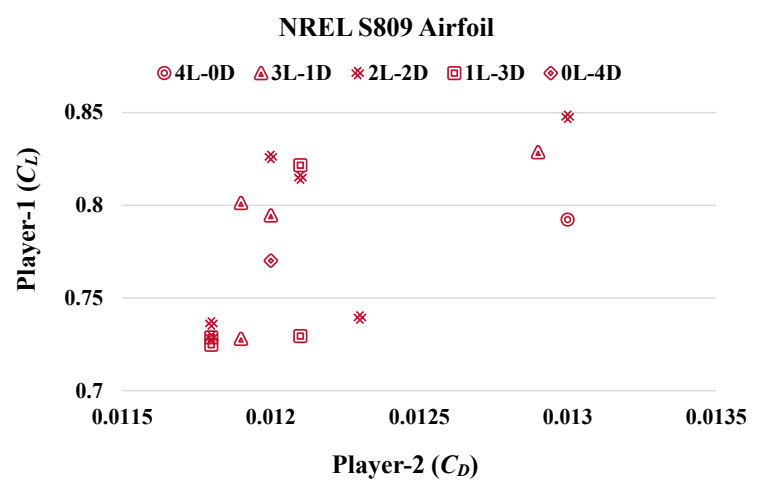

(a)

Locust Wing Profile

○4L-0D $\triangle 3 L-1 D * 2 L-2 D \quad$ 回L-3D $\odot 0 L-4 D$

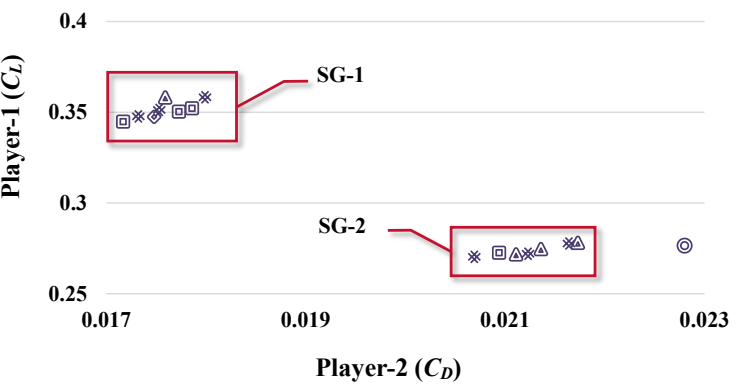

(b)

Fig. 5. Scatter plot of the players 1 and 2 forming Nash equilibria set for (a) an NREL S809 airfoil, (b) locust wing cross-section profile at $20 \%$ spanwise chord.

A series of two-dimensional CFD analysis is carried out to evaluate the airfoils in a steady, viscous, and incompressible air flow at $R e=7000\left(0.6 \mathrm{~ms}^{-1}\right)$ and $\alpha=2^{\circ}$. Prior to the optimization of our bioinspired airfoil, the proposed procedure is validated with respect to the literature [25] using an NREL S809 airfoil shown in Figure 5(a). Here, a clear relationship is established among the points within the Nash equilibria set, where highest possible $C_{L}$ values are obtained by the combinations whose common DVs are mostly assigned to player-1, and vice-versa, which is in line with the results reported previously.

Similarly, the points forming the Nash equilibria set for an optimized locust airfoil at $20 \%$ spanwise chord are concentrated in two distinct regions SG-1 and SG-2. This is graphically represented in Figure 5(b). Also, similar to a conventional smooth-surfaced airfoil, maximum $C_{L}$ or minimum $C_{D}$ is achieved by the strategies whose common DVs are mostly assigned to the players 1 or 2 , respectively. This validates our proposed bioinspired optimization process conducted on the corrugated locust airfoil.

\section{A. Aerodynamic Forces}

The obtained force coefficients of the original locust airfoils in this study are already aerodynamically superior to the available literature [11]. The gliding ratio is at least 6 times greater than the peer reported data. This difference is mainly due to the deeply curved forefoils (extended top crest vertical position) of the peer models causing a sharp raise in their drag coefficients.

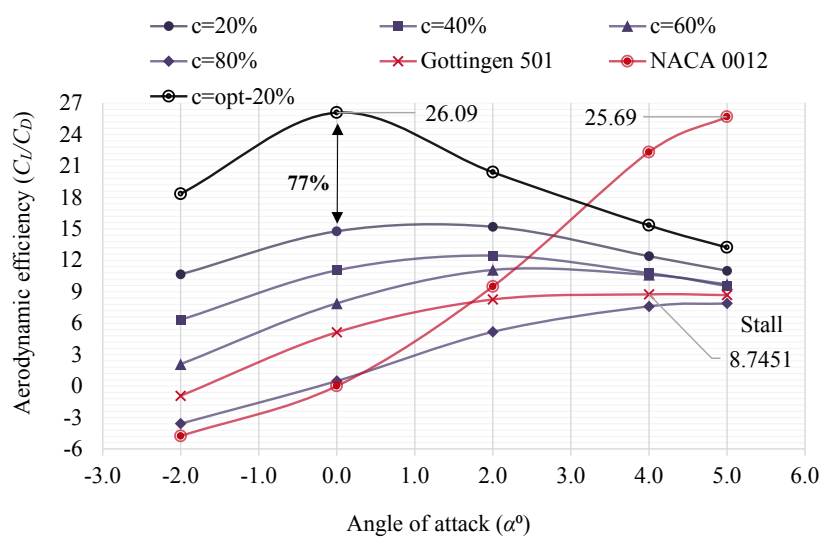

Fig. 6. Illustration of the aerodynamic efficiency for locust airfoils at $20 \%$, $40 \%, 60 \%$, and $80 \%$ spanwise chord, subjected to a $R e=7000$ flow at different angles of attack.

On the contrary, aerodynamic forces that we determined are rather comparable to a conventional smooth-surfaced airfoil operating at a low $R e$. For instance, peak aerodynamic efficiency of the $\mathrm{c}=20 \%$ airfoil shown in Figure 6 is determined as 15.2 whereas, a conventional slow-flight Gottingen 501 airfoil with a maximum thickness of $12.8 \%$ at $30 \%$ chord and maximum camber of $6.3 \%$ at $50 \%$ chord produces a $C_{L} / C_{D}$ of 8.7 at $R e=50,000$ and $\alpha=4^{\circ}$, or a NACA 0012 delivers a maximum efficiency of 25.6 at $\alpha=5^{\circ}$ and $R e=50,000$ [35] shown in Figure 6.

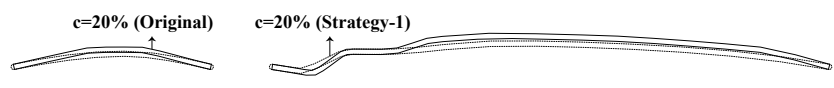

(a)
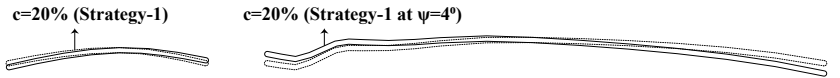

(b)

Fig. 7. Schematic representation of the optimized locust wing crosssection profile at $20 \%$ spanwise chord. (a) original and the strategy-1 airfoil superimposed, (b) strategy-1 airfoils at fore-hindfoil phase shift $\left(\psi=4^{\circ}\right)$.

Furthermore, considering the optimized airfoil ( $\mathrm{c}=\mathrm{opt}-$ $20 \%$ ) performance, the quantitative analysis proves a peak improvement of $77 \%$ in lift-drag ratio $\left(\eta_{a}\right)$ at $\alpha=0^{\circ}$. As mentioned earlier, the airfoils developed as a result of strategies with maximum common DVs assigned to each player, delivers optimum performance with respect to that player only. Hence, the airfoils with maximum $C_{L}$ exhibited longer suction portion and greater thickness, and the minimum drag airfoils are better streamlined with a limited thickness and sharper tips. However, a design trade-off strategy must 
be established where the airfoil produces maximum lift at minimum drag. This is best achieved by the airfoil resulted from strategy-1 DV assignment belonging to group 1L-3D in the SG-1 region, producing a lift coefficient of $C_{L}=0.2781$ and a drag coefficient of $C_{D}=0.0171$. This airfoil with a focus on lower drag is selected due to the fact that its $C_{D}$ is minimized by $25 \%$ with respect to the original airfoil, whereas the highest $C_{L}$ obtained by any strategy does not exceed $4 \%$ as a result of the reduction in PARSEC parameters.

Figure 7(a) illustrates a superimposition of the original and strategy-1 airfoils. It is clear that the hindfoil common DVs in millimeter $\left(u_{b}=1.464, v_{b}=0.231, v_{t}=1.184\right)$ are reduced by $25 \%\left(u_{b}=1.098, v_{b}=0.1738, v_{t}=0.888\right)$ restricting the curves and suction regions, instead streamlining (profiling) the external geometry. However, Figure 7(b) illustrates a combination of the proposed and a conventional bioinspired wing optimization methodology [15], [17], [18] introducing a fore-hindfoil phase shift of $\psi=4^{\circ}$. Addition of the conventional scheme contributed an almost $61 \%$ of the enhancement in aerodynamic performance of the optimized airfoil by increasing $\eta_{a}=16.2$ to $\eta_{a}=26.09$ at $\alpha=0^{\circ}$, and producing a $C_{L}$ and $C_{D}$ of 0.3154 and 0.0120 , respectively.

\section{B. Pressure $\left(C_{P}\right)$ Distribution}

Finally, pressure distribution over the airfoil is analyzed to validate our digitized geometry aerodynamically. As seen

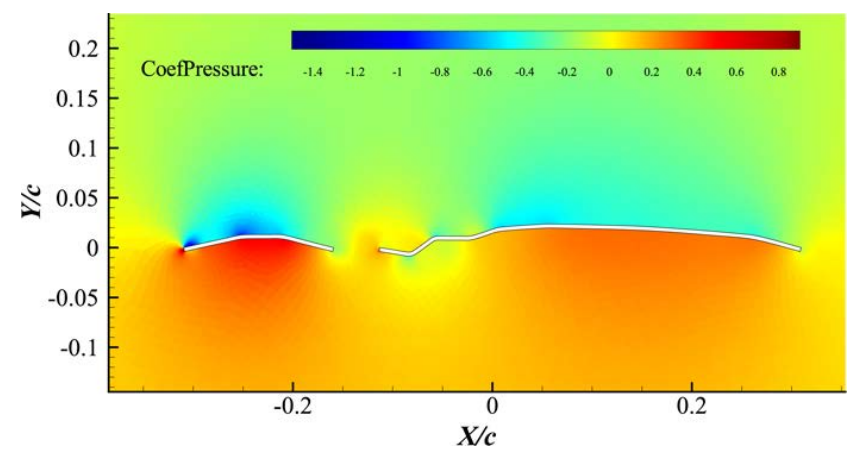

(a)

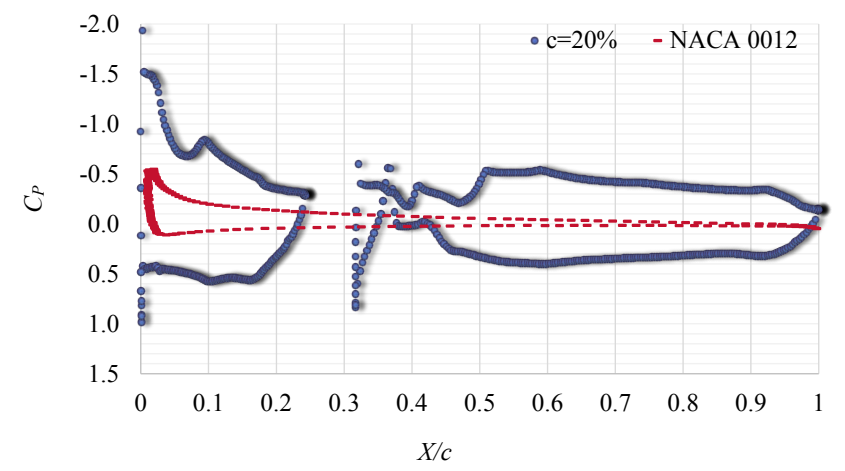

(b)

Fig. 8. Illustration of the converged pressure coefficient distribution for locust wing profile at $20 \%$ spanwise chord, subjected to a flow at $R e=$ 7000 and $\alpha=2^{\circ}$. (a) $C_{P}$ contours, and (b) $C_{P}$ distribution across chord.

in Figures 8(a) and 9(a), Bernoulli's principle is satisfied, where the lower and higher value pressure gradients spread over the top and bottom of the airfoil surface, respectively.

The pressure distribution over an airfoil greatly depends on geometrical parameters such as wing thickness, camber, chord, and aspect ratio. We quantify $C_{P}$ distribution over the airfoil chord with the help of a two-dimensional scatter plot shown in Figure 8(b) to facilitate classification of sections of the airfoil surface and their value-specific influence on the air flow. A precise analysis of the Figure 8(b) clearly categorizes a number of regions on the fore- and hindfoil geometry that are locally and globally influencing the flow field. These are; (a) profile tip that is one of the most crucial regions. A squared tip (represented in almost every research on corrugated airfoils) causes the formation of an extremely unstructured mesh at this critical region which in turn delays the solution convergence. Also, it creates

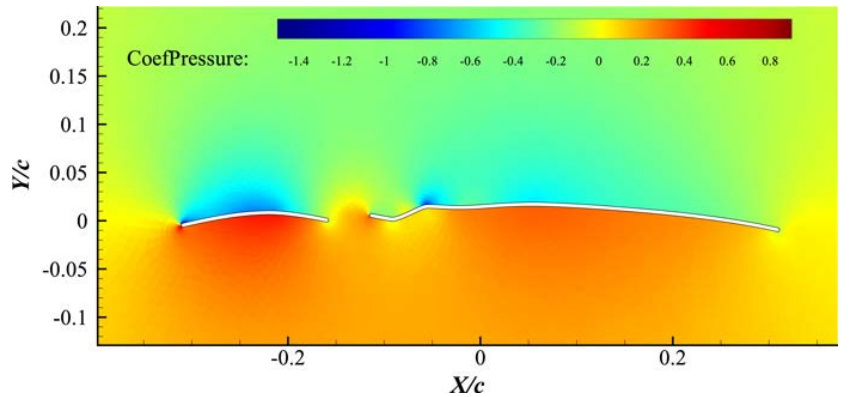

(a)

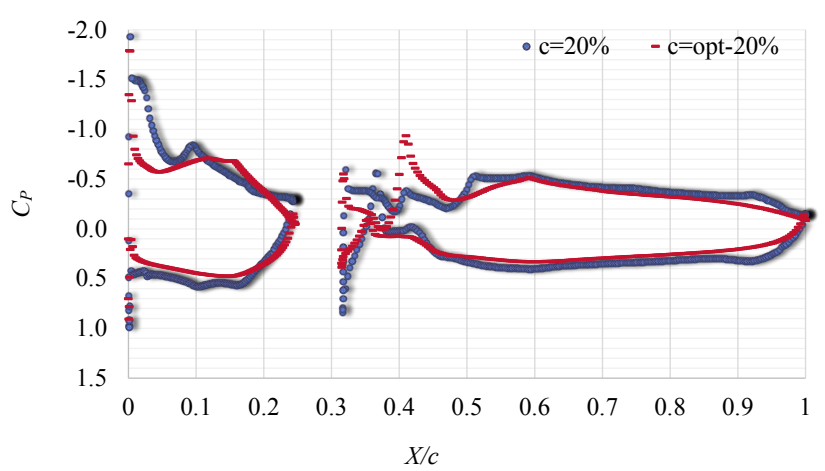

(b)

Fig. 9. Illustration of the converged pressure coefficient distribution for the optimized locust wing profile (c=opt-20\%), subjected to a flow at $R e=$ 7000 and $\alpha=2^{\circ}$. (a) $C_{P}$ contours, and (b) $C_{P}$ distribution across chord.

an immediate flow separation as a result of its right angle of incidence on the fluid flow. Similarly, for the NACA airfoil, this is the region where the pressure drops swiftly cutting through the inflow. (b) Fore- and hindfoil LE and TE angles of incidence strongly influencing the upwash, flow separation, and reattachment of the corrugation slopes. However, the hindfoil TE is of greater importance as it is responsible in producing a downwash which directly influences the overall lift generated, and should this region be well optimized, the lift will be maximized with a minimum increase in drag similar to conventional high-lift devices. (c) Fore- and hindfoil phasing $(\psi)$, also discussed earlier as a major energy-recovery factor that can be made beneficial 


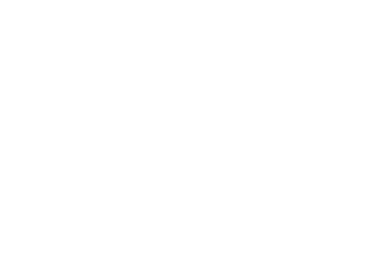

Vorticity Magnitude

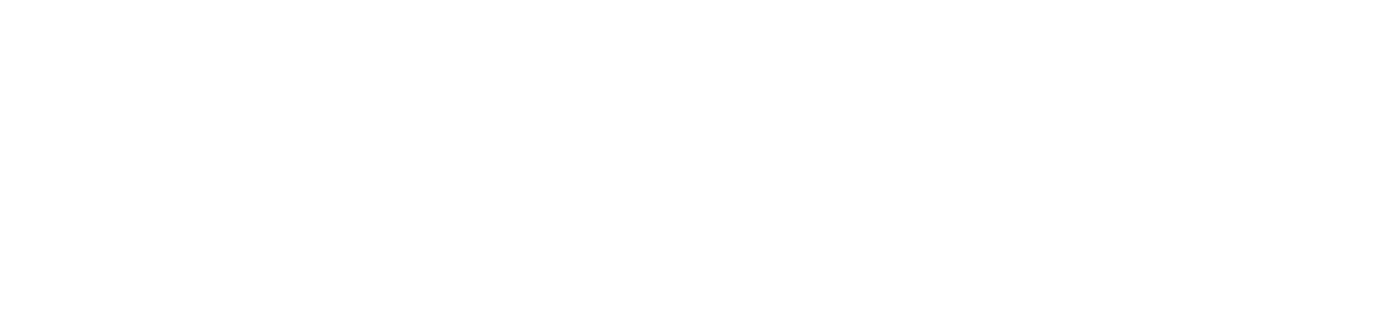

(a)

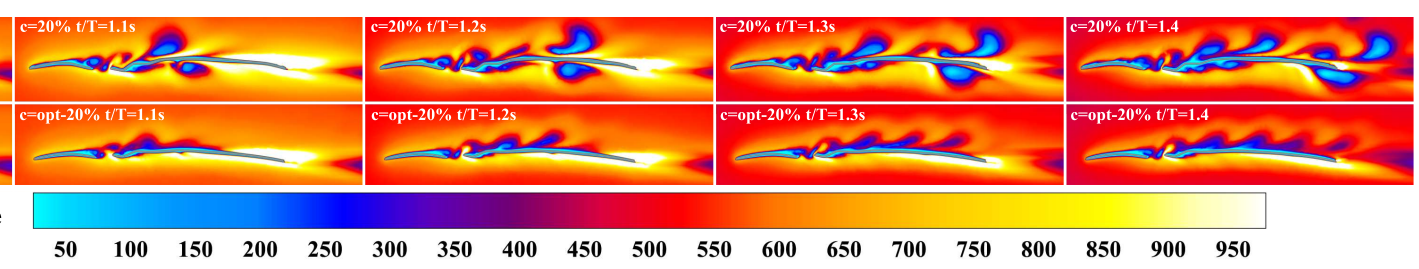

(b)

Fig. 10. Graphical representation of the vorticity contour sequence at $R e=7000$ and $\alpha=5^{\circ}$ for timestamps $1.0 \mathrm{~s}$ to $1.4 \mathrm{~s}$. (a) the airfoils at $40 \%, 60 \%$, and $80 \%$ spanwise chord, and (b) the original $(\mathrm{c}=20 \%)$ and optimized $(\mathrm{c}=\mathrm{opt}-20 \%)$ airfoil at $20 \%$ spanwise chord.

when tested in various configurations. These factors are taken into consideration while designing $\mathrm{c}=$ opt- $20 \%$ shown in Figure 9(b), where the pressure is evidently distributed more smoothly across the airfoil chord in comparison to the original pressure distribution (blue plots). This is mainly due to the $4^{\circ}$ shift in fore-hindfoil angle of attack, and profiling of the airfoil corrugations.

\section{Vortex Interactions}

Here, the cross-section profiles of the locust wing at $20 \%, 40 \%, 60 \%$, and $80 \%$ spanwise chord along with the optimized airfoil (c=opt-20\%) are subjected to a transient, viscous, and an incompressible air flow at $R e=7000(0.6$ $\mathrm{ms}^{-1}$ ) and $\alpha=5^{\circ}$. Only during the vorticity analysis, the viscosity is defined by a four-equation shear stress transport (SST) $k-\omega$ turbulence model developed by Menter et al. [36]. To run the simulations fully turbulent accommodating the transitional $R e$ regime, a low-Reynolds number correction is introduced using the low-Re coefficient $\left(\alpha^{*}\right)$ that dampens the eddy viscosity.

We shall assume the relative fore- hindfoil angle of attack defined as airfoil phasing $\left(\psi=0^{\circ}\right)$ for the original airfoils. This creates 2 major vortical interactions within the LE \& TE observed as;

- The passing Leading-Edge Vortex (LEV) of the forefoils mildly interacting with the LE of the hindfoils that induces either a suction or a separation depending on the hindfoil LE angle of incidence. Induced suction generates further thrust as in the case of airfoils closer to the wing-root $(c=20 \%, c=40 \%)$, whereas flow separation retards the body illustrated in $\mathrm{c}=80 \%$. These interactions remain mild due to a zero fore-hindfoil phase shift assumption.

- Also, the passing Trailing-Edge Vortex (TEV) of the forefoils interact with the LE of the hindfoils rather severely due to a stronger TEV shedding. This induces mainly flow separation exhibited by all three airfoils shown, however, the energy in these vortices can harnessed to achieve insect-level aerodynamic efficiencies.

It is evident from the above observations that the airfoil phasing $(\psi)$, and the LE \& TE angles of incidence play a crucial role in the process of energy restoration and hence, endured flight. Adjustment of these parameters is a common energy-conservation practice for tandem winged fliers [15], and locust is certainly no exception. Therefore, identifying such attributes for performance improvement shall bolster the foundations (parameterization) of our optimization process.

Furthermore, considering the optimized airfoil flow field, we observe a mild formation of LEV due to a $25 \%$ reduction in forefoil top crest vertical position $\left(v_{t}\right)$, which in turn couples with the strong TEV shedding in order to induce a flow suction on the hindfoil nose portion, further increasing the overall flow velocity and lift shown in Figure 10(b). It is clear that the optimized airfoil's hindfoil TEV are almost eliminated, and its LEV shedding remains fairly uniform throughout. These are some of the bolstering factors behind the steep $(25 \%)$ drag reduction in our optimized airfoil.

\section{CONCLUSIONS}

This paper presents a 2D computational fluid dynamic analysis on the aerodynamic characteristics of locust wing cross-section profiles in gliding mode. The profiles are subjected to a bioinspired mathematical optimization scheme based on PARSEC parameterization and Nash-Genetic algorithms which results in a set of Nash equilibria points facilitating the establishment of a balanced trade-off strategy to obtain higher $(10 \%)$ aerodynamic efficiency. However, addition of conventional methodologies such as fore-hindwing phase shift provided further efficiency by enhancing the performance $(77 \%)$. Thus, proving that locusts' endured gliding capability originates from their; fore-hindwing, and peer-wing vortex interactions as a result of their tandem wing and swarm behavior. Ultimately, the extension of this 
research shall focus on the $3 \mathrm{D}$ reconstruction and prototyping of the locust wing for a comprehensive PIV wind tunnel testing.

\section{ACKNOWLEDGMENT}

This work is supported by EU Horizon 2020 projects STEP2DYNA [Grant No.691154]; ULTRACEPT [Grant No.778062]; and partially supported by the National Natural Science Foundation of China [Grant No.91648203]; and the Natural Science Foundation of Hubei Province [Grant No.2018CFB431].

\section{REFERENCES}

[1] J. W. S. Rayleigh, "The soaring of birds," Nature, vol. 27, pp. 534$535,1883$.

[2] G. Walker, "The flapping flight of birds," J. Royal Aeronautical Society, vol. 29, no. 179, pp. 590-594, 1925.

[3] C. P. Ellington, "The aerodynamics of hovering insect flight. III. Kinematics," Philosophical Transactions of the Royal Society of London. Series B, Biological Sciences, vol. 305, no. 1122, pp. 41-78, 1984.

[4] M. W. Lorenz, "Migration and trans-Atlantic flight of locusts," $J$. Quaternary International, vol. 196, no. 2, pp. 4-12, 2009.

[5] H. Isakhani, N. Aouf, O. Kechagias-Stamatis, and J. F. Whidborne, "A furcated visual collision avoidance system for an autonomous micro robot," IEEE Trans. Cognitive and Developmental Systems, pp. 1-11, 2018.

[6] S. Yue and F. C. Rind, "Redundant neural vision systems-competing for collision recognition roles," IEEE Trans. Autonomous Mental Development, vol. 5, no. 2, pp. 173-186, 2013.

[7] — , "Near range path navigation using LGMD visual neural networks," in Proc. Int. Conf. Computer Science and Information Technology, 2009, pp. 105-109.

[8] T. Weis-Fogh, "Biology and physics of locust flight. II. Flight performance of the desert locust (Schistocerca gregaria)," Philosophical Transactions of the Royal Society of London. Series B, Biological Sciences, vol. 239, no. 667, pp. 459-510, 1956.

[9] M. Cloupeau, J. F. Devillers, and D. Devezeaux, "Direct measurements of instantaneous lift in desert locust; comparison with Jensen's experiments on detached wings," J. Experimental Biology, vol. 80, pp. $1-15,1979$.

[10] P. J. Simmons, F. C. Rind, and R. D. Santer, "Escapes with and without preparation: The neuroethology of visual startle in locusts," J. Insect Physiology, vol. 56, no. 8, pp. 876-883, 2010.

[11] J. Xiang, J. Du, D. Li, and K. Liu, "Aerodynamic performance of the locust wing in gliding mode at low reynolds number," J. Bionic Engineering, vol. 13, no. 2, pp. 249-260, 2016.

[12] S. M. Walker, A. L. R. Thomas, and G. K. Taylor, "Deformable wing kinematics in the desert locust: how and why do camber, twist and topography vary through the stroke?" J. R. Soc. Interface, vol. 6, no. 38, pp. 735-747, 2009.

[13] M. Kovač, Wassim-Hraiz, O. Fauria, J. Zufferey, and D. Floreano, "The EPFL jumpglider: A hybrid jumping and gliding robot with rigid or folding wings," IEEE Int. Conf. Robotics and Biomimetics, pp. 1503-1508, 2011 .

[14] P. Henningsson, D. Michaelis, T. Nakata, D. Schanz, R. Geisler, A. Schröder, and R. J. Bomphrey, "The complex aerodynamic footprint of desert locusts revealed by large-volume tomographic particle image velocimetry," J. R. Soc. Interface, vol. 12, no. 108, pp. 1-11, 2015.

[15] D. E. Rival, G. Hass, and C. Tropea, "Recovery of energy from leading- and trailing-edge vortices in tandem-airfoil configurations," J. Aircraft, vol. 48, no. 1-2, pp. 203-211, 2011.

[16] H. Gopalan and A. Povitsky, "Lift enhancement of flapping airfoils by generalized pitching motion," J. Aircraft, vol. 47, no. 6, pp. 18841897, 2010.

[17] P. Henningsson and R. J. Bomphrey, "Time-varying span efficiency through the wingbeat of desert locusts," J. R. Soc. Interface, vol. 9, no. 71, pp. 1177-1186, 2012.

[18] T. Q. Le, T. V. Truong, S. H. Park, T. Q. Truong, J. H. Ko, H. C. Park, and D. Byun, "Improvement of the aerodynamic performance by wing flexibility and elytra-hind wing interaction of a beetle during forward flight," J. R. Soc. Interface, vol. 10, no. 85, pp. 1-15, 2013.
[19] J. A. Samareh, "A survey of shape parameterization techniques," in CEAS/AIAA/ICASE/NASA Langely Int. Forum on Aeroelasticity and Structural Dynamics, Williamsburg, VA, 1999, pp. 333-344.

[20] H. Sobieczky, "Geometry generator for CFD and applied aerodynamics," in Courses and Lecture International, 1997, pp. 137-158.

[21] — , "Parametric airfoils and wings," in Notes on Numerical Fluid Mechanics, 1998, vol. 68, pp. 71-88.

[22] D. E. Goldberg, Genetic Algorithms in Search, Optimization, and Machine Learning. Addison-Wesley Professional, 1989.

[23] N. Srinivas and K. Deb, "Multiobjective optimisation using nondominated sorting in genetic algorithms," Evolutionary Computation, vol. 2, no. 3, pp. 221-248, 1994.

[24] J. Nash, "Non-Cooperative Games," Annals of Mathematics, vol. 54, no. 2, pp. 286-295, 1951.

[25] P. Della Vecchia, E. Daniele, and E. D'Amato, "An airfoil shape optimization technique coupling PARSEC parameterization and evolutionary algorithm," J. Aerospace Science and Technology, vol. 32, no. 1, pp. 103-110, 2014.

[26] J. D. Anderson, Fundamentals of Aerodynamics, 5th ed. McGrawHill, 2011.

[27] H. Hu and M. Tamai, "Bioinspired corrugated airfoil at low Reynolds numbers," J. Aircraft, vol. 45, no. 6, pp. 2068-2077, 2008.

[28] P. Spalart and S. Allmaras, "A one-equation turbulence model for aerodynamic flows," Recherche Aerospatiale, vol. 1, pp. 5-21, 1992.

[29] D. E. Levy and A. Seifert, "Simplified dragonfly airfoil aerodynamics at Reynolds numbers below 8000," Physics of Fluids, vol. 21, no. 7, 2009.

[30] T. Basar and G. J. Olsder, Dynamic noncooperative Game theory. Academic Press, New York., 1995, vol. 23, no. 2.

[31] R. L. Haupt and S. E. Haupt, Practical Genetic Algorithms. WileyInterscience, 1998.

[32] E. D'Amato, E. Daniele, L. Mallozzi, and G. Petrone, "Equilibrium strategies via GA to Stackelberg Games under multiple follower's best reply," Int. J. Intelligent Systems, vol. 27, pp. 74-85, 2012.

[33] E. D'Amato, E. Daniele, L. Mallozzi, G. Petrone, and S. Tancredi, "A hierarchical multimodal hybrid Stackelberg-Nash GA for a leader with multiple followers game," in Dynamics of Information Systems: Mathematical Foundations, 2012, vol. 20, pp. 267-280.

[34] D. Fudenberg and J. Tirole, Game Theory. The MIT Press, 1991.

[35] M. Drela and H. Youngren, "XFOIL: An analysis and design system for low Reynolds number airfoils," in Mueller T.J. (eds) Low Reynolds Number Aerodynamics. Lecture Notes in Engineering. Springer, Berlin, Heidelberg, 1989, pp. 1-12.

[36] F. Menter, M. Kuntz, and R. Langtry, "Ten years of industrial experience with the SST turbulence model," Turbulence, Heat and Mass Transfer, pp. 625-632, 2003. 